Recebido: 21-01-2021 | Aprovado: 07-07-2021 | DOI: https://doi.org/10.23882/MJ2268

\title{
O uso de dispositivos eletrónicos por crianças dos 0 aos 5 anos de idade
}

\section{The use of electronic devices by children from 0 to 5 years of age}

\author{
Bruno Rocha, Universidade do Algarve, Portugal (bmp.rocha@gmail.com) \\ Cristina Nunes, Centro de Investigação em Psicologia (CIP/UAL), \\ Universidade do Algarve, Portugal (csnunes@ualg.pt)
}

\section{Resumo:}

Enquadramento: O tempo que as crianças passam com ecrãs é cada vez maior, sendo uma preocupação pelas implicações no seu desenvolvimento e saúde.

Objetivos: Este estudo teve como objetivo conhecer a utilização dos dispositivos eletrónicos multimédia por crianças dos 0 aos 5 anos em Portugal.

Metodologia: Foi realizado um estudo quantitativo, descritivo-correlacional e transversal com 547 pais de crianças com idades entre os 0 e os 5 anos, aos quais foi aplicado um questionário online.

Resultados: Os resultados mostraram que $82 \%$ das crianças veem televisão em média 85 minutos por dia e $57 \%$ utilizam o tablet ou smartphone 51 minutos por dia. $\mathrm{O}$ uso aumenta com a idade das crianças e está negativamente associado à escolaridade dos pais.

Conclusão: O uso de dispositivos eletrónicos por parte das crianças é superior ao recomendado pela Organização Mundial de Saúde. É importante que seja reduzido o tempo de ecrã para valores mais próximos dos recomendados e que o uso dos mesmos seja controlado pelos pais com conteúdos educativos e adequados à idade.

Palavras-Chave: Pré-escolares, Pais, Multimédia

\begin{abstract}
:
Background: Children's screen time is increasing and is a cause of concern for the implications in their development and health.

Aim: This study aimed to understand the use of electronic multimedia devices by children from 0 to 5 years old in Portugal.

Methodology: A quantitative, descriptive-correlational and cross-sectional study was conducted with 547 parents of children aged from 0 to 5 years, to whom an online questionnaire was applied.

Results: The results showed that $82 \%$ of children watch television on average 85 minutes per day and $57 \%$ use the tablet or smartphone 51 minutes per day. The use of these devices increases with children's age and is negatively associated with the parents' level of education.

Conclusion: The use of electronic devices by children is higher than that recommended by the World Health Organization. Guidelines are discussed to minimize the impact of the use of electronic devices on health and development.
\end{abstract}

Keywords: Preschool students, Parents, Multimedia 


\section{Introdução}

A forma como os dispositivos eletrónicos e a internet têm vindo a integrar o nosso estilo de vida constitui uma preocupação para os profissionais da saúde e investigadores pelas suas repercussões para a saúde e desenvolvimento das crianças. Os dispositivos eletrónicos fazem parte do dia-a-dia das crianças. Para além da televisão interativa e dos canais infantis temáticos, verifica-se cada vez mais uma maior utilização dos pequenos dispositivos eletrónicos com ligação à internet, tais como o smartphone ou o tablet (Brito \& Ramos, 2017; Sociedade Brasileira de Pediatria (SBP), 2016).

Nikken e Schols (2015), num estudo realizado na Holanda com crianças até aos 7 anos, dos 10 tipos de dispositivos multimédia estudados, identificou que os 4 mais utilizados foram a televisão, os de touchscreen, as consolas de jogos e os computadores.

O uso dos dispositivos eletrónicos digitais deveria ser limitado e proporcional à idade e etapas de desenvolvimento cognitivo e social das crianças e adolescentes (SBP, 2006). Tanto a Organização Mundial de Saúde (OMS), como a Academia Americana de Pediatria (AAP), como a SBP, desaconselham a utilização de dispositivos eletrónicos até aos 2 anos de idade (AAP Council on Communications and Media, 2016; SBP, 2006; World Health Organization (WHO), 2019), referindo que apesar de alguns benefícios, são mais os prejuízos daí resultantes.

A AAP indica ainda que, dos 18 meses aos 2 anos, caso os pais desejem iniciar a introdução dos media digitais, deverão fazê-lo sempre com supervisão, sendo sempre eles a escolher e a controlar as aplicações, evitando que as crianças usem os dispositivos tecnológicos de forma autónoma. Refere ainda que, antes dos 18 meses, o único tipo de exposição a ecrãs tolerado é a vídeo-chamada (AAP Council on Communications and Media, 2016). A vídeo-chamada tende a ser aceite pelos pais, mesmo quando estes restringem a utilização de dispositivos eletrónicos (McClure et al., 2015). Dos 2 aos 5 anos não é recomendada exposição a ecrãs por mais do que uma hora diária (AAP Council on Communications and Media, 2016; WHO, 2019) e os conteúdos devem ser educativos, adaptados à idade das crianças e não violentos (Rocha $\&$ Nunes, 2020).

Apesar das recomendações referidas acima, é um facto que o uso de ecrãs em crianças é comum (Howe et al., 2017), sendo a televisão o aparelho com maior uso (Adisak et al., 2018; Ponte et al., 2017), e outros dispositivos de touchscreen maioritariamente utilizados para visualização de vídeos (Kabali et al., 2015; Ponte et al., 2017). Cerca de metade das crianças com 2 anos acedem à internet ocasionalmente, aumentando o número, frequência e a autonomia com a idade (AAP, 2018; Kabali et al., 2015). Por exemplo, na Nova Zelândia, 76\% das crianças com menos de 2 anos usavam a televisão em média cerca de 21 min por dia e $16 \%$ o touchscreen (Howe et al., 2017). No Reino Unido, dos 6 aos 36 meses, 75.2\% das crianças usavam o touschreen, em média 24.45 min por dia (Bedford et al., 2016). Na Holanda, nas crianças menores de 7 anos, a média de uso de televisão foi de 51 min e cerca de 11 min nos restantes dispositivos (Nikken \& Schols, 2015). Em Portugal, as crianças com idades compreendidas entre os 3 e os 5 anos, passam em média 154 min por dia (95\% CI: $149.51-$ 158.91) em frente a ecrãs (Rodrigues et al., 2020).

Face ao aumento globalizado do uso de dispositivos eletrónicos no dia-a-dia das pessoas, e ao impacto que esse fenómeno pode trazer para a saúde e desenvolvimento das crianças, este estudo tem como objetivo conhecer de que forma os dispositivos eletrónicos multimédia são usados por crianças dos 0 aos 5 anos em Portugal, sendo norteado pelas seguintes questões de investigação: Quais os dispositivos eletrónicos mais usados pelas crianças dos 0 aos 5 anos em Portugal? Qual a duração desse uso e qual a preocupação dos pais com os conteúdos visionados? 


\section{Método}

Trata-se de um estudo quantitativo, descritivocorrelacional e transversal. A amostra foi constituída por 574 pais de crianças residentes em Portugal, dos 0 aos 5 anos de idade, inclusive.

Os dados foram recolhidos com um questionário com 27 itens de auto-resposta, adaptado do estudo de Nikken e Schols (2015) no que respeita à forma como era questionado o uso de dispositivos eletrónicos, nomeadamente se tem o respetivo dispositivo eletrónico em casa, se o utiliza, quanto tempo o utiliza e se o tem no seu quarto. A maioria das questões foi elaborada como variável dicotómica ( $\operatorname{sim} /$ não), havendo 3 perguntas na forma de escala de Likert, nomeadamente se o seu filho(a) mais novo usa o smartphone ou o tablet na companhia de um adulto, se se preocupa que os conteúdos desses dispositivos sejam adequados à idade e se são educativos. No que respeita à questão de num dia normal, quanto tempo o seu filho(a) mais novo usa o dispositivo eletrónico, a resposta é fechada para horas e minutos. Antes da sua aplicação, o questionário foi sujeito à apreciação qualitativa por parte de um painel de 5 participantes com os critérios da amostra, para aferir erros e dificuldades.

Os itens incluíram perguntas de caracterização sociodemográfica sobre os pais (sexo e idade, estado civil, escolaridade e situação laboral), caracterização sociodemográfica das crianças (idade e sexo), envolvimento parental no uso dos dispositivos eletrónicos (presença do adulto, preocupação com adequação dos conteúdos), e perguntas relacionadas com a frequência do uso pelas crianças (dispositivos de que dispõem, acessibilidade e periodicidade do uso).

A seleção da amostra foi por conveniência, utilizando o método de bola de neve. O primeiro autor solicitou aos participantes o contacto de outros potenciais participantes do seu conhecimento. A recolha de dados inicial contou com 640 respondentes, dos quais 66 foram excluídos por não estarem integrados na faixa etária em estudo. A recolha de dados decorreu on-line durante o mês de maio de 2020. No caso de os pais terem mais de um filho, as perguntas foram sempre orientadas para que a resposta fosse em função do seu filho mais novo. Ainda que o estudo tenha ocorrido durante o período de início da pandemia Covid-19, quando as crianças se encontravam em confinamento domiciliário predispondo um maior uso de dispositivos eletrónicos por parte das mesmas, as perguntas recordavam frequentemente que se referia à utilização habitual antes do confinamento. $\mathrm{O}$ estudo foi aprovado pela Comissão de Ética da ARS Algarve I.P. (proc $n^{0} 4 / 2020$ ).

Os dados foram analisados utilizando o software SPSS v25 (IBM SPSS, 2017). Em primeiro lugar foram realizadas análises descritivas das variáveis sociodemográficas, apresentando o tamanho da amostra, percentagens, média e desvio padrão, conforme o tipo de variável. Em segundo lugar, foram apresentadas as variáveis relativas ao uso de dispositivos eletrónicos de forma descritiva, assim como a associação destas com as variáveis sociodemográficas através do uso do coeficiente $r$ de Pearson para correlação. A ANOVA univariada e o teste de qui-quadrado foram utilizados para a comparação entre grupos.

\section{Resultados}

1 - Características sociodemográficas dos participantes

A idade dos pais variou entre os 21 e os 50 anos $(M=35.8 ; D P=4.95)$. Dos 574 participantes $86.8 \%$ eram mães e $13.2 \%$ pais. Quanto ao estado civil estavam casados ou em união de facto $84.5 \%$, solteiros $11.5 \%$, divorciados $3.8 \%$ e viúvos $0.2 \%$. No que respeita à escolaridade, $67.6 \%$ concluíram o ensino superior, 27.4\% o ensino secundário, $4.2 \%$ o $3^{\circ}$ ciclo e $0.9 \%$ o $2^{\circ}$ ciclo. Acerca da atividade laboral, $10.6 \%$ estavam desempregados.

As crianças tinham em média 24.5 meses de idade $(D P=15.0), 51.9 \%$ eram rapazes e $48.1 \%$ raparigas.

\section{2 - Uso dos dispositivos eletrónicos}

$\mathrm{Na}$ Tabela 1 apresentamos o uso dos dispositivos eletrónicos. 
Tabela 1

Análise do uso dos vários dispositivos eletrónicos mais frequentes dos 0 aos 5 anos

\begin{tabular}{|c|c|c|c|}
\hline Dispositivos eletrónicos disponíveis em casa & Sim & & \\
\hline Televisão & $99.8 \%$ & & \\
\hline Consolas de jogos & $41.5 \%$ & & \\
\hline Computador & $95.3 \%$ & & \\
\hline Smartphone ou Tablet & $97.2 \%$ & & \\
\hline \multicolumn{4}{|l|}{ Dispositivos eletrónicos usados pela criança } \\
\hline Televisão & $81.2 \%$ & & \\
\hline Consolas de jogos & $5.6 \%$ & & \\
\hline Computador & $5.1 \%$ & & \\
\hline Smartphone ou Tablet & $56.8 \%$ & & \\
\hline \multicolumn{4}{|l|}{ Dispositivos eletrónicos existentes no quarto da criança } \\
\hline Televisão & $14.4 \%$ & & \\
\hline Consolas de jogos & $3.1 \%$ & & \\
\hline Computador & $0 \%$ & & \\
\hline Smartphone ou Tablet & $6.1 \%$ & & \\
\hline Tempo diário de uso dos dispositivos eletrónicos (min por dia) & $M$ & $D P$ & $n$ \\
\hline Televisão & 84.78 & 77.47 & 466 \\
\hline Consolas de jogos & 33.94 & 32.01 & 32 \\
\hline Computador & 37.72 & 48.05 & 29 \\
\hline Smartphone ou Tablet & 50.79 & 51.62 & 326 \\
\hline
\end{tabular}

No que respeita à relação do tempo de ecrã com outras variáveis, observámos que existe uma relação positiva entre a idade da criança e o tempo de uso da televisão $(r=.19 ; p=.000)$ e de dispositivos de touchscreen $(r=.20 ; p=.000)$, isto é, o tempo de uso destes dispositivos multimédia, aumenta com a idade. Observámos também que quando a escolaridade dos pais é menor, aumenta tempo de o uso da televisão pelas crianças $(r=-.11 ; p=.012)$ e dos dispositivos de touchscreen $(r=-.25 ; p=.000)$ (Tabela 2$)$.

Não observámos diferenças significativas entre o tempo de ecrã e outras variáveis sociodemográficas (sexo da criança, sexo e idade dos pais, estado civil e situação laboral.

\section{Tabela 2}

Relação entre o tempo diário de uso dos dispositivos eletrónicos em crianças dos 0 aos 5 anos e as variáveis sociodemográficas.

\begin{tabular}{lc}
\hline \multicolumn{2}{c}{ Tempo diário de uso dos dispositivos eletrónicos } \\
\hline & $r$ \\
\hline Idade dos pais & Televisão \\
\hline Escolaridade & -.05 \\
\hline Idade da criança & $\mathbf{- . 1 1 *}$ \\
\hline & $\mathbf{. 1 9}^{* *}$ \\
\hline Idade dos pais & .04 \\
\hline Escolaridade & .22 \\
\hline Idade da criança & .18 \\
\hline & Computador \\
\hline Idade dos pais & -.01 \\
\hline Escolaridade & .00 \\
\hline Idade da criança & .13 \\
\hline & Touchscreen \\
\hline Idade dos pais & .02 \\
\hline Escolaridade & $\mathbf{- 2 5}$ \\
\hline Idade da criança & $\mathbf{. 2 0}$ \\
\hline Nota: ** $p<.01 ; ~ p<.05$ & \\
\hline
\end{tabular}


Tabela 3

Uso de dispositivos eletrónicos em crianças com mais e com menos de 24 meses de idade

\begin{tabular}{lcccc}
\hline \multicolumn{1}{c}{ Idade } & $\begin{array}{c}0-23 \mathrm{~m} \\
\% / M(D P)\end{array}$ & $\begin{array}{c}24-60 \mathrm{~m} \\
\% / M(D P)\end{array}$ & $\chi^{2} / F$ & \\
& & & & \\
\hline Dispositivos usados & & & & \\
\hline Televisão & $68.5 \%$ & $93 \%$ & 56.19 & .000 \\
\hline Consolas de jogos & $1.4 \%$ & $9.4 \%$ & 17.19 & .000 \\
\hline Computador & $1.8 \%$ & $8.1 \%$ & 11.64 & .001 \\
\hline Smartphone ou Tablet & $30.1 \%$ & $81.5 \%$ & 154.70 & .000 \\
\hline Uso dos dispositivos (min por dia) & & & & .000 \\
\hline Televisão & $67.85(70.10)$ & $96.34(80.20)$ & 15.68 & .455 \\
\hline Consolas de jogos & $22.50(28.72)$ & $35.57(32.69)$ & 0.57 & .317 \\
\hline Computador & $17.80(24.27)$ & $41.88(51.03)$ & 1.04 & .000 \\
\hline Smartphone ou Tablet & $33.39(31.18)$ & $56.74(54.95)$ & 13.15 & \\
\hline
\end{tabular}

A televisão e os dispositivos de touchscreen são os mais usados pelas crianças. Observámos que depois dos 2 anos o uso de dispositivos eletrónicos aumenta consideravelmente, existindo uma diferença significativa entre as crianças mais novas e as crianças mais velhas em especial nos dispositivos de touchscreen $\left(\chi^{2}=154.70 ; p=.000\right)$ e na televisão $\left(\chi^{2}=56.19 ; p=.000\right)$ (Tabela 3$)$.

Quanto ao uso de dispositivos de touchscreen, dos 574 participantes, $56.8 \%$ afirmam que o seu filho mais novo usa algum dispositivo de touchscreen, nomeadamente smarphone ou tablet (Figura 1).

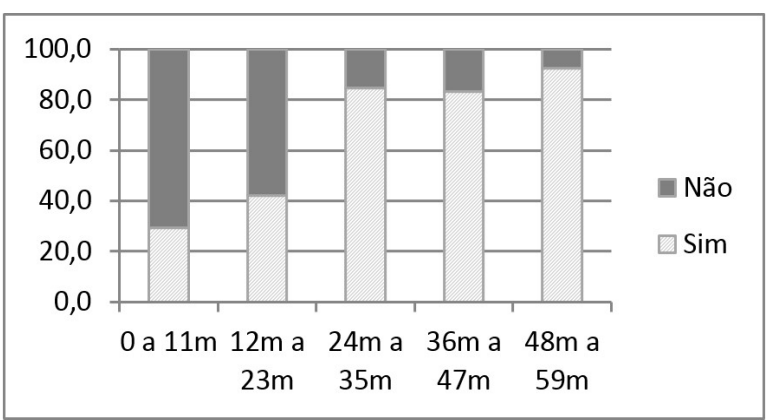

Figura 1. Uso de dispositivos de touchscreen por faixa etária

Dos 343 participantes que referiram que os seus filhos usavam dispositivos de touchscreen, 69.1\% fazem-no praticamente sempre na presença de um adulto, não se verificando relação com a idade das crianças $(r=-.07 ; p=.198)$. A preocupação com o tipo de conteúdos existe para $83.4 \%$ dos pais, também ela sem relação com a idade das crianças $(r=-.05 ; p=.325)$. Por fim, $56.6 \%$ dos pais preocupam-se que o teor dos conteúdos seja educativo, existindo uma relação negativa com a idade das crianças $(r=-.11, p=.051)$.

\section{Discussão}

No nosso estudo praticamente todas as crianças utilizam algum dispositivo eletrónico em casa, o que vai ao encontro a outros estudos, embora com valores ligeiramente mais elevados que os mostrados por Nikken e Schols (2015). Pensamos que os cinco anos que decorreram entre os estudos pode explicar estas diferenças esse aumento, assim como o atual cenário pandémico em que os dados foram recolhidos. A tendência do aumento da frequência no acesso à tecnologia digital ao longo dos anos é referido por Ponte et al. (2017).

Em idades inferiores a 5 anos, os dispositivos eletrónicos mais usados por crianças são a televisão $(81.2 \%)$, logo seguidos pelos dispositivos de touchscreen (56.8\%). Nesta faixa etária, o computador e as consolas de jogos têm uma expressão 
bastante bem menos relevante, sendo cerca de $5 \%$. Nikken e Schols (2015) tinham observado que, até aos 7 anos, os tipos de dispositivos mais utilizados eram a televisão, o touchscreen, o computador e a consola de jogos. No presente estudo os dispositivos mais presentes na vida das crianças são a televisão e os dispositivos de touchscreen. Esta diferença pode ser devida a que no nosso estudo as crianças são mais novas e as consolas e computadores são dispositivos mais complexos do ponto de vista do utilizador.

As crianças até aos 5 anos tendencialmente não têm aparelhos multimédia no seu quarto, tendo maior expressão a televisão com $14.4 \%$, valor semelhante aos $16 \%$ referidos por Nikken e Schols (2015). A televisão é o dispositivo eletrónico mais usado pelas crianças, que visionam em média 85 min por dia $(D P=77 \mathrm{~min}$.), seguido dos smartphones e tablets com um uso em média de 51 min por dia $(D P=51 \mathrm{~min}$.$) . Estes resultados mostram as$ mesmas tendências de outros estudos onde a televisão é o principal dispositivo, seguido do touchscreen (Adisak et al., 2018; Kabali et al., 2015; Ponte et al., 2017). Tal como Nikken e Schols (2015) salientaram, também o tempo médio de uso dos dispositivos eletrónicos tende a aumentar com a idade. $\mathrm{O}$ elevado desvio padrão também é uma característica nos vários estudos, o que demonstra que, embora 0 uso dos dispositivos eletrónicos tenha um padrão crescente com a idade nas crianças, tem também uma grande variabilidade no padrão de comportamento entre elas, havendo dentro de dada faixa etária, crianças que usam muito menos ou muito mais que a média dos seus pares (Howe et al., 2017; Nikken \& Schols, 2015).

Nikken e Schols (2015) mostraram uma média de uso de televisão de $51 \mathrm{~min}$ e cerca de $11 \mathrm{~min}$ nos restantes dispositivos, bastante inferior aos 85 min de televisão e aos 51 min de touchscreen reportados no presente estudo. A diferença temporal entre os estudos é possivelmente uma das variáveis que contribuem para este aumento tão significativo. Mas pode também ser devida a diferenças culturais e estilos de vida, que levem provavelmente a que as crianças holandesas estejam expostas a menos tempo de ecrã que as portuguesas.

As crianças portuguesas com menos de dois anos também têm um tempo de exposição superior aos ecrãs, em que $68.5 \%$ vê televisão em média 67.85 min por dia e $31.5 \%$ usa dispositivos de touchscreen 33.39 min por dia. Isto é três vezes mais televisão e duas vezes mais de dispositivos de touchscreen que as crianças nos estudos de Howe et al. (2017) e Bedford et al. (2016).

Tal como Howe et al. (2017), também observámos um aumento significativo no uso dos dispositivos a partir dos 24 meses de idade. Quando comparamos estes resultados com as recomendações da AAP e da OMS, verifica-se que o tempo de ecrã das crianças está muito acima do aceitável, e que quando comparado com estudos anteriores os comportamentos têm vindo a piorar. Este estudo mostra um aumento muito acentuado no que respeita ao tempo médio diário do uso de dispositivos eletrónicos. A OMS e a AAP sugerem que crianças depois dos 24 meses não assistam a mais do que 1 hora diária de aparelhos multimédia, neste estudo, a televisão é usada em média $96.34 \min (D P=80.20)$ por dia e os aparelhos de touchscreen entre os $56.74 \mathrm{~min}(D P=$ 54,95). Estes valores referidos atrás são apresentados de forma isolada, podendo ainda ser cumulativos entre os vários dispositivos. Quando comparamos estes dados aos resultados de Rodrigues et al. (2020) em Portugal, com uma média de 154 min por dia no uso total de ecrãs em crianças dos 3 aos 5 anos, verificamos que a soma do uso da televisão e do touchscreen do presente estudo, na faixa etária dos 2 aos 5 anos, é de 153 min por dia, valor este praticamente igual. Estes resultados são preocupantes pois o aumento do tempo de ecrã está relacionado com um menor bemestar psicossocial e problemas de comportamento, assim como com problemas de conduta e atenção no futuro (Rocha \& Nunes, 2020; Zhao et al., 2018). 
Em média, 80\% das crianças usavam os dispositivos eletrónicos na maior parte das vezes na companhia de um adulto, havendo assim 20\% das crianças que o fazem sem este acompanhamento. Este resultado é menos positivo, pois como refere Mendelsohn et al. (2010) a supervisão e acompanhamento parental é importante e facilita as aprendizagens.

A grande maioria dos pais (95.6\%) preocupavase com a adequação dos conteúdos à idade e com o seu carater educativo (89.8\%), sendo estas preocupações independentes da idade da criança. Estes dois resultados são positivos, pois são aspetos que contribuem para minimizar o impacto negativo da exposição aos ecrãs (AAP Council on Communications and Media, 2016).

Tomopoulos et al. (2007) afirmam que o aumento do tempo a conteúdos não educacionais leva a menos tempo para o ensino e leitura, interferindo negativamente com o seu desenvolvimento. No âmbito da promoção da saúde infantil, Silva et al. (2018) reforçam a importância das políticas e estratégias de intervenção da promoção de comportamentos de vida saudáveis. Wu et al. (2014) mostraram que os problemas de temperamento e comportamento das crianças tendem a estar relacionados com um maior tempo de ecrã, especialmente se o conteúdo for não educativo ou antissocial.

Tal como em estudos anteriores (Howe et al., 2017; Nunes et al., 2016), a escolaridade dos pais relacionou-se significativa e negativamente com o tempo de uso da televisão e do touchscreen, sendo que quanto menor a escolaridade mais o tempo de exposição dos filhos a ecrãs. Outros fatores preditores descritos na literatura, como o desemprego ou a existência de televisão no quarto da criança, não mostraram relação significativa neste estudo com o tempo de uso dos dispositivos referidos acima (Howe et al., 2017).

No que respeita às principais limitações deste estudo, salientamos as seguintes. A amostra foi recolhida na sua totalidade on line, por resposta voluntária a um questionário que foi partilhado nas redes sociais e divulgado em sites para pais de crianças, o que de certa forma acaba por restringir as respostas a um grupo de pessoas utilizadoras destas plataformas. A maioria dos participantes tem uma elevada escolaridade, o que revela uma amostra heterogénea. Ainda que tivesse sido pedido especificamente para que as respostas fossem acerca do comportamento habitual e anterior das crianças, a recolha de dados durante o período de confinamento social obrigatório pode ter levado a que a perceção dos pais sobre o uso fosse diferente ao que responderiam caso no caso de nunca ter havido um período de confinamento pela à pandemia Covid-19.

Este estudo traz dados mais recentes sobre o uso de dispositivos eletrónicos por crianças portuguesas dos 0 aos 5 anos de idade. Existindo uma relação do uso de dispositivos eletrónicos e o desenvolvimento infantil, esta informação pode ser importante para o desenvolvimento de projetos de intervenção comunitária. Estudos e intervenções futuras podem aprofundar o contexto em que os dispositivos eletrónicos são usados pelas crianças, assim como elaborar políticas de saúde que visem a redução e o uso saudável dos ecrãs em crianças.

\section{Conclusão}

Conclui-se que os dispositivos eletrónicos mais usados pelas crianças dos 0 aos 5 anos são a televisão e os dispositivos de touchscreen. Os restantes dispositivos multimédia não apresentam expressão relevante. Face aos estudos anteriores analisados, existe claramente um aumento do uso de dispositivos eletrónicos por parte de crianças pequenas. As crianças passam claramente mais tempo no uso de ecrãs do que aquele recomendado pela OMS e pela AAP. A televisão é o dispositivo mais popular, contudo o uso de dispositivos de touchscreen tem vindo a aumentar ao longo do tempo.

Os pais, na sua maioria, procuram que os conteúdos sejam educativos e adequados à idade das cri- 
anças. No entanto observámos que muitos pais negligenciam o impacto negativo do uso de dispositivos eletrónicos. As crianças cujos pais têm menor escolaridade estão expostas aos ecrãs durante mais tempo, o que mostra a importância do trabalho de aconselhamento às famílias nesta matéria.

É urgente a promoção de políticas, campanhas e atividades promotoras de saúde em duas grandes frentes: primeiro reduzir o tempo de ecrã nas cri- anças para valores mais próximos dos recomendados, segundo, promover que o uso de dispositivos eletrónicos seja controlado pelos pais, escolhendo conteúdos educativos e adequados à idade.

\section{Conflitos de interesse}

Os autores não têm conflitos de interesse a declarar. Não houve qualquer apoio ou subsídio financeiro a declarar para a realização do estudo.

\section{Referências}

American Academy of Pediatrics (2018). Media and Children Communication Toolkit. Retrieved from https://www.aap.org/en-us/advocacy-and-policy/aap-health-initiatives/Pages/Media-andChildren.aspx

American Academy of Pediatrics Council on Communications and Media (2016). Media and Young Minds. Pediatrics, 138, e20162591. https://doi.org/10.1542/peds.2016-2591

Adisak, P., Chiranuwat, S., Pongtong, P., \& Sakda Arj-Ong, V. (2018). ICT exposure in children younger than 2 years: rates, associated factors, and health outcomes. Journal of the Medical Association of Thailand, 101, e345. http://www.jmatonline.com/index.php/jmat/article/view/8883

Bedford, R., Saez of Urabain, I. R., Cheung. C. H. M., Karmiloff-Smith, A., \& Smith, T. J. (2016). Toddlers' fine motor milestone achievement is associated with early touchscreen scrolling. Frontiers in Psychology, 7, el 108. https://doi.org/10.3389/fpsyg.2016.01108

Brito, R., \& Ramos, A. (2017). Digital technology in family environment: A case of children from 0 to 6. In: International Symposium on Computers in Education (SIIE) (pp. 1-4). IEEE. https://doi.org/10.1109/SIIE.2017.8259673Howe, A. S., Heath, A. M., Lawrence, J., Galland, B. C., Gray, A. R., Taylor, B. J., Sayers, R., \& Taylor, R. W. (2017). Parenting style and family type, but not child temperament, are associated with television viewing time in children at two years of age. Plos ONE, 12(12), 1-16. https://doi.org/10.1371/journal.pone.0188558

Kabali, H. K., Irigoyen, M. M., Nunez-Davis R., Budacki. J. G., Mohanty, S. H., Leister, K. P., \& Bonner R. L. Jr. (2015, December). Exposure and use of mobile media devices by young children. Pediatrics, 136, 1044-1050. https://doi.org/10.1542/peds.2015-2151. 
McClure, E. R., Chentsova-Dutton, Y. E., Barr, R. F., Holochwost, S. J., \& Parrott, W. G. (2015). Facetime doesn't count: video chat as an exception to media restrictions for infants and toddlers. International Journal of Child-Computer Interaction, 6, 1-6. https://doi.org/10.1016/j.ijcci.2016. 02.002

Mendelsohn, A. L., Brockmeyer, C. A., Dreyer B. P., Fierman, A. H., Berkule-Silberman, S. B., \& Tomopoulos, S. (2010). Do verbal interactions with infants during electronic media exposure mitigate adverse impacts on their language development as toddlers? Infant and Child Development, 19, 577-593. https://doi.org/10.1002/icd.711

Nikken, P., \& Schols, M. (2015). How and Why Parents Guide the Media Use of Young Children. Journal of Child and Family Studies, 24, 3423-3435. https://doi.org/10.1007/s10826-015-0144-4

Nunes, C., Matos, F., \& Costa, E. (2016). Qualidade de vida e bem-estar nas crianças em Albufeira. Relatório técnico. Faro: Universidade do Algarve.

Ponte, C., Simões, J. A., Baptista, S., Jorge, A., \& Castro, T. S. (2017). Crescendo entre ecrãs: usos de meios eletrónicos por crianças (3-8 Anos). Entidade Reguladora para a Comunicação Social. https://www.erc.pt/documentos/Crescendoentreecras/mobile/index.html\#p=1

Rocha, B., \& Nunes, C. (2020). Benefits and damages of the use of touchscreen devices for the health and development of children from 0 to 5 years of age - A systematic review. Psicologia: Reflexão e Crítica, 33, e24 . https://doi.org/10.1186/s41155-020-00163-8

Rodrigues, D., Gama, A., Machado-Rodrigues, A., Nogueira, H., Silva, M., Marques, V., \& Padez, C. (2020). Social inequalities in traditional and emerging screen devices among Portuguese children: a cross-sectional study. BMC Public Health, 20, e902. https://doi.org/10.1186/s12889-02009026-4

Silva, E., Simões, P., Macedo, M., Duarte, J., \& Silva, D. (2018). Perceção parental sobre hábitos e qualidade do sono das crianças em idade pré-escolar. Revista de Enfermagem Referência, 17, 63-72. https://doi.org/10.12707/RIV17103

Sociedade Brasileira de Pediatria (2016). Manual de orientação: saúde de crianças e adolescentes na era digital. Sociedade Brasileira de Pediatria.

http://www.sbp.com.br/fileadmin/user_upload/2016/11/19166d-MOrient-Saude-Crian-e-Adolesc.pdf

Tomopoulos, S., Valdez, P. T., Dreyer, B. P., Fierman, A. H., Berkule, S. B., Kuhn, M., \& Mendelsohn, A. L. (2007). Is exposure to media intended for preschool children associated with less parent-child shared reading aloud and teaching activities? Ambulatory Pediatrics, 7, 18-24. https://doi.org/10.1016/j.ambp.2006.10.005

World Health Organization (2019). Guidelines on physical activity, sedentary behavior and sleep for children under 5 years of age. World Health Organization. http://www.who.int/iris/ handle/10665/311664

Wu, C. S., Fowler, C., Lam, W. Y., Wong, H. T., Wong, C. H., \& Loke, A. Y. (2014). Parenting approaches and digital technology use of preschool age children in a Chinese community. Italian Journal of Pediatrics, 40, e44. https://doi.org/10.1186/1824-7288-40-44

Zhao, J., Zhang, Y., Jiang, F., Ip, P., KaWing Ho, F., Zhang, Y., \& Huang, H. (2018). Excessive screen time and psychosocial well-being: the mediating role of body mass index, sleep duration, and parent-child interaction. JAMA Pediatrics, 202, 7-62. https://doi.org/10.1016/j.jpeds.2018.06.029 